International Journal of Medical Anesthesiology 2021; 4(2): 171-175

\begin{tabular}{l|c} 
Medical Anesthesiology \\
of
\end{tabular}

\author{
Dr. Alka Shah, Dr. Rahul Ramchandani, Dr. Pringal Gohil and \\ Dr. Chandra Chandrasekharan
}

DNB Anaesthesia,

Department of Anaesthesia,

GMERS Medical College Sola,

Ahmedabad, Gujarat, India

Dr. Pringal Gohil

$3^{\text {rd }}$ Year Resident Anaesthesia,

Department of Anaesthesia,

GMERS Medical College Sola,

Ahmedabad, Gujarat, India

Dr. Chandra Chandrasekharan $2^{\text {nd }}$ Year Resident Anaesthesia, Department of Anaesthesia, GMERS Medical College Sola, Ahmedabad, Gujarat, India

Corresponding Author: Dr. Pringal Gohil $3^{\text {rd }}$ Year Resident Anaesthesia, Department of Anaesthesia, GMERS Medical College Sola, Ahmedabad, Gujarat, India

\section{DOI: https://doi.org/10.33545/26643766.2021.v4.i2c.248}

\section{Abstract}

Background: Inguinal hernia repair is a common surgical procedure. Chronic pain occurs in 5-10\% after the inguinal hernia repair. This study is aimed to evaluate post-operative analgesia efficacy of ultrasound guided transverse abdominal plane (TAP) block in unilateral hernia surgery.

Aims and Objective: The present study aimed to evaluate the post-operative pain scoring at $1 / 2$, $2,4,6,12,18$ and 24 hours duration on basis of pain intensity using Visual Analogue Scale (VAS). Total requirement of analgesic drug in first 24 hours. To compare time for first rescue analgesia.

Material and Method: A total of $80(n=80)$ patients of ASA I and II grade in the age group of 20 to 65 years undergoing unilateral hernia surgery. They were randomized into two groups. Lumbar puncture was done at L3-L4 space through a standard $25 \mathrm{G}$ quincke spinal needle in sitting position of patient. Drug $0.5 \%$ hyperbaric bupivacaine $3.5 \mathrm{ml}$ was administered intrathecally as a single dose in all patient. Study group: Patients received TAP block with Ropivacaine $(0.5 \%) 20 \mathrm{ml}$ after completion of surgery. Control group: Patients not received TAP block and was given injection diclofenac on demand for postoperative analgesia. The primary outcome was measured as patient-reported pain intensity using VAS.

Results: VAS score was significantly lower in study group post-operatively at all time intervals $(\mathrm{P}<0.0001)$. The 24 -h diclofenac consumption was significantly less in study group compared to control group $(93.7 \pm 32.88 \mathrm{mg}$ vs. $204.3 \pm 44.89 \mathrm{mg}, \mathrm{P}<0.0001)$. Time for first rescue analgesia was significantly more in study group as compared to control group (635.66 \pm 312.2 minutes vs. $210 \pm 140.7$ minutes, $\mathrm{p}<0.0001$ )

Conclusion: TAP block provided excellent post-operative analgesia in the first $24 \mathrm{~h}$.

Keywords: TAP block, unilateral inguinal hernia, post-operative analgesia, ropivacaine, diclofenac

\section{Introduction}

Inguinal hernia repair is a common surgical procedure ${ }^{[1]}$. The incidence is reported as 11/10,000 in persons between 16 and 24 years of age, 200/10,000 in persons more than 75 years of age ${ }^{[2]}$. Chronic pain occurs in $5-10 \%$ after the inguinal hernia repair that creates an important problem ${ }^{[3]}$.

The postoperative management of pain following hernia surgery can pose a challenge to anaesthesia providers. A significant part of pain after hernia surgery is caused by the abdominal wall incision and conducted by the lower intercostal nerves and iliohypogastric and ilioinguinal nerves (i.e. T11 to L1) ${ }^{[4]}$.

Rafi in 2015 introduced the local anaesthetics into the transversus abdominis plane (TAP) via the triangle of Petit, it is possible to block the sensory nerves of the anterior abdominal wall before they leave this plane and pierce the musculature to innervate the entire anterior abdominal wall (T7 to L1) ${ }^{[5,6]}$.

Traditional pharmacological pain management with opioids and non-steroidal antiinflammatory drugs (NSAIDS) in post hernia surgery has been reported to cause inadequate pain control.

Hebbard et al (2007) ${ }^{[7]}$ have subsequently described an ultrasound-guided (USG) approach 
to the TAP block. Real-time ultrasound provides reliable imaging of the three muscular layers of the anterolateral abdominal wall and assessment of correct needle placement and local anesthetic injection thus potentially increasing the success rate and safety of the TAP block compared to the landmark technique.

We hypothesised that the analgesic efficacy of TAP block performed under ultrasound would provide a better postoperative analgesia with fewer complications, in comparison to our standard practice of opioids and NSAIDS.

\section{Methods}

This prospective randomized non blinded interventional study was conducted on patients posted for elective unilateral hernia surgery at a tertiary care teaching hospital in India, after obtaining ethical clearance.

The study period was 1 year from 15-05-2019 till 14-052020. The exclusion criteria included patients who did not give consent, those with allergy or sensitivity to local anaesthetic agents, patients having bleeding disorders or on anticoagulants, those having a history of treatment for a chronic pain condition and were on daily analgesics for more than 4 weeks, those with basal metabolic index $>35$ $\mathrm{kg} / \mathrm{m} 2$ or the American Society of Anesthesiologists (ASA) physical status >II, presence of neurological deficit, psychiatric illness and those with infection at the site of injection. This study included 20-65 years patients of ASA physical status I or II and posted for unilateral inguinal hernia. A written informed consent was taken from all the patients. The participants were allocated randomly into two groups of 40 each by using computer-based random number generator.

All patient were fasted overnight prior to the scheduled day of operation. All surgeries were carried out under spinal anaesthesia. On arrival to operation theatre, following insertion of $18 \mathrm{G}$ venous cannula, all patients were received $15 \mathrm{ml} / \mathrm{kg}$ of Ringer's Lactate solution before spinal anaesthesia. Standard monitors like electrocardiography (ECG), Noninvasive Blood Pressure (NIBP) monitoring and Pulse Oximetry were attached and the baseline parameters was recorded. Lumbar puncture was done at L3-L4 space through a standard $25 \mathrm{G}$ quincke spinal needle in sitting position of patient. Drug $0.5 \%$ hyperbaric bupivacaine 3.5 $\mathrm{ml}$ was administered intrathecally as a single dose in all patient. All patients were received oxygen $5 \mathrm{~L} / \mathrm{min}$ via a face mask throughout the procedure after approximating them to the surgical position.

Sensory block was assessed by sterile pin prick method in the midaxillary line on both sides of chest. Immediately after sensory block assessment, motor block was assessed using a modified Bromage scale. The highest dermatomal level of sensory block and recovery times of both sensory and motor block were recorded. Recovery time for the sensory block is defined as two dermatome regression of anaesthesia from the maximal level. Motor block duration is the time to return to grade 0 on the modified Bromage scale. Highest dermatomal level of sensory block after completion of surgery was recorded.

Hypotension (defined by a decrease in MAP below 20\% of baseline or systolic pressure $<90 \mathrm{~mm} \mathrm{Hg}$ ) was treated with incremental doses of mephentermine $3.0 \mathrm{mg}$ i.v. and additional lactated Ringer's solution as appropriate. Bradycardia (HR $<50$ beats/minute) will be treated with atropine $0.6 \mathrm{mg}$ i.v. To avoid masking of respiratory depression by administering supplemental oxygen, respiratory depression is defined as a respiratory rate $<12$ breathes/ minutes.

After completion of surgery TAP block will be performed under all sterile precaution on the same side of surgery with ultrasonographic guidance with a Sono Site portable ultrasound device and a linear 6-13 $\mathrm{MHz}$ ultrasound transducer. Once the external oblique abdominal muscle (EOAM), internal oblique abdominal muscle (IOAM) and transverse abdominal muscle (TAM) were visualized at the level of the anterior axillary line between the 12th rib and the iliac crest, the puncture area and the ultrasound probe were prepared in a sterile manner. After identification of the neuro-facial plane between IOAM and TAM, block was performed with $18 \mathrm{G}$ Touhy needle. The needle was directed to approach the TAP with "in plane" ultrasound guided technique. Once the tip of the needle was placed in the space between the IOAM and TAM, inj. Ropivacaine $(0.5 \%) 20 \mathrm{ml}$ was injected after negative aspiration. The drug was seen spreading in TAP as a dark oval shape.

But in control group, patients did not receive TAP block for analgesia postoperatively. These patients were managed by injection Diclofenac as per hospital protocol. Postoperative pain was assessed by using the visual analogue scale in both groups. Patients with a VAS score of 3 or more received injection diclofenac sodium $75 \mathrm{mg}$ intramuscularly. Duration of postoperative analgesia was considered after completion of surgery upto first requirement of analgesia. Total dose of inj. diclofenac in $24 \mathrm{hr}$. was noted. Vital signs (respiratory rate, $\mathrm{SpO} 2$, heart rate, blood pressure), VAS scores, cumulative dose of diclofenac at $30 \mathrm{~min}, 2,4,6,12$, 18 , and $24 \mathrm{hr}$. postoperatively were noted. Complications of TAP block were noted.

\section{Statistical analysis}

When primary comparison is between means of two samples in experimental design, the statistical formula for sample size calculation was

\section{$\mathrm{N}>2(\mathrm{Z} \alpha+\mathrm{Z} \beta)^{2} \times \mathrm{SD}^{2}$ $\mathrm{d}^{2}$}

Alpha $(\alpha)$ Error - $0.05[\mathrm{Z} \alpha=1.96]$

Beta $(\beta)$ Error $-0.20[Z \beta=0.842]$, (power of the study 90\%) S.D. = Estimated standard deviation (S.D.) of pain score (vas score) $=2$

$\mathrm{d}=$ Estimated difference of means of pain score (vas score) in two groups $=2$

Standardised difference $=$ difference between two mean in two treatment group devided by population standard deviation $=\frac{4-2}{2}=1$

According to this formula based nomogram, sample size for proposed study was:

$\mathrm{N}=40$ patients were taken in each group.

Control group $(n=40)$. Patient not received TAP block. Patient received injection diclofenac.

Study group $(n=40)$ patient received usg guided TAP block with injection ropivacaine. 
Both the groups were given injection diclofenac on demand as per VAS score for post-operative analgesia as per institute protocol for routine surgery.

Statistical analysis was performed using the Graphpad prism version 6.0.7 software (GraphPad Software, Inc. USA). In our study sample size was 40 in each group and equal in both group so we used unpaired student t-test for analysis [41]. Demographic data were analysed using unpaired student"s t-test. According to Shapiro-wilk normality test, ttest was used for homogenous variables such as time to first requirement of diclofenac(duration of analgesia), and total requirement of diclofenac. Measurements of pain scores were analyzed at each time interval performed using the unpaired t-test ${ }^{[12]}$. Vitals were also analysed by student $t-$ test. A difference with significant level (p- value< 0.05 ) was considered statistically significant.

\section{Results}

This prospective study was carried out in 80 ASA I and II patients, who underwent open unilateral inguinal hernioplasty. They were randomly divided into two different groups.

Study group: Patients received TAP block with Ropivacaine $(0.5 \%) 20 \mathrm{ml}$.

Control group: Patients not received TAP block and was given injection diclofenac on

demand for postoperative analgesia as per institute protocol for routine surgery.

Table 1: Demographic Data

\begin{tabular}{|c|c|c|c|c|}
\hline \multicolumn{2}{|c|}{ Group } & Study & Control & P Value* \\
\hline \multicolumn{2}{|c|}{ Age (YEARS)* } & $46.38 \pm 11.66$ & $50.53 \pm 11.7$ & .1161 \\
\hline \multicolumn{2}{|c|}{ Weight $(\mathrm{KG})^{*}$} & $62.52 \pm 6.62$ & $61.125 \pm 5.03$ & .2919 \\
\hline \multicolumn{2}{|c|}{ Height $(\mathrm{CM})^{*}$} & $167.6 \pm 6.14$ & $167.9 \pm 4.48$ & .8035 \\
\hline \multirow{2}{*}{ Sex } & Male & 37 & 38 & \multirow{2}{*}{.9054} \\
\hline & Female & 3 & 2 & \\
\hline
\end{tabular}

(*using unpaired student $t$ test)

$\mathrm{P}>0.05$, Values are mean $\pm \mathrm{SD}$ and numbers

Patients were in age group of 20 to 65 years in both group. Both groups were comparable in terms of age, weight and height. No significant difference was observed between two group in terms of demographic data.

Table 2: Vas Score In The Study And Control Groups

\begin{tabular}{|c|c|c|c|c|c|c|c|c|}
\hline Group & Preop erativ e & $\mathbf{3 0 m i n}$ & $\mathbf{2 h r}$ & $\mathbf{4 h r}$ & $\mathbf{6 h r}$ & $\mathbf{1 2 h \mathbf { h r }}$ & $\mathbf{1 8 h \mathbf { h r }}$ & $\mathbf{2 4 h \mathbf { h }}$ \\
\hline \multirow{2}{*}{ Study } & 0 & $0.4 \pm$ & $0.87 \pm$ & $1.35 \pm$ & $2.05 \pm$ & $2.22 \pm$ & $1.6 \pm$ & $2.1 \pm$ \\
& 0 & 0.5 & 0.40 & 0.69 & 1.29 & 1.09 & 0.8 & 0.9 \\
\hline \multirow{2}{*}{ Control } & 0 & $1.07 \pm$ & $2.45 \pm$ & $2.9 \pm$ & $2.4 \pm$ & $3.8 \pm$ & $3.12 \pm$ & $3.7 \pm$ \\
& 0.65 & 1.08 & 1.17 & 0.7 & 0.76 & 0.68 & 0.81 \\
\hline P value* & 0 & $<0.0001$ & $<0.0001$ & $<0.0001$ & $<0.04$ & $<0.0001$ & $<0.0001$ & $<0.0001$ \\
\hline
\end{tabular}

(*using unpaired student ${ }^{\text {ee }} \mathrm{t}$ test)

$\mathrm{P}^{*}<0.05$, Significant difference in both group

VAS score was significantly higher in control group as compared to the study group at all the time.

Table 3: Time For First Dose Of Rescue Analgesia

\begin{tabular}{|c|c|}
\hline Groups & Time For First Dose of Rescue Analgesia In Minute \\
\hline Study & $635.66 \pm 312.2$ Minutes \\
\hline Control & $210 \pm 140.7$ Minutes \\
\hline P Value* & $\begin{array}{c}<0.0001 \\
\text { (Extremely Statistically Significant Difference) }\end{array}$ \\
\hline
\end{tabular}

First dose of rescue analgesia required in study group was at $635.66 \pm 312.2$ minutes and in control group was $210 \pm 140.7$ min which was statistically highly significant.
Table 4: Total Diclofenec Consumption In $24 \mathrm{Hr}$

\begin{tabular}{|c|c|}
\hline Group & Diclfenac dose in mg \\
\hline Study & $93.7 \pm 32.88 \mathrm{mg}$ \\
\hline Control & $204.3 \pm 44.89 \mathrm{mg}$ \\
\hline P value* & $<0.0001$ \\
\hline
\end{tabular}

(*using unpaired student's t-test) $\mathrm{P}^{*}<0.0001$ statistically very highly significant

Total dose of DICLOFENAC consumption in study group was $93.7 \pm 32.88 \mathrm{mg}$ and in control group it was $204.3 \pm 44.89$ mg, which showed that DICLOFENAC consumption was significantly decreased in study group.

Table 5: Cumulative Consumption of Diclofenac

\begin{tabular}{|c|c|c|c|c|c|c|c|c|}
\hline Group & Preoperative & 30min & 2hr & 4hr & 6hr & 12hr & 18hr & 24hr \\
\hline Study & 0 & 0 & 0 & $5.6 \pm 20$ & $26.25 \pm 36.22$ & $31.87 \pm 37.54$ & $7.5 \pm 22.78$ & $22.5 \pm 34.8$ \\
\hline Control & 0 & 0 & $25.25 \pm 36.22$ & $33.75 \pm 37.87$ & $7.5 \pm 22.7$ & $65.62 \pm 25.11$ & $13.12 \pm 28.86$ & $58.12 \pm 31.71$ \\
\hline P value $*$ & 0 & 0 & 0 & $<0.0001$ & 0.0069 & $<0.0001$ & 0.3367 & $<0.0001$ \\
\hline
\end{tabular}

(*using unpaired student ${ }^{*} \mathrm{~s} \mathrm{t}$ test) $* \mathrm{P}$ value $<0.05$ shows statistically significant difference in both groups 
Cumulative Diclofenac Consumption

\begin{tabular}{|c|c|c|c|c|c|c|c|c|c|}
\hline & Group & Preoperative & 30mi n & $\mathbf{2 h r}$ & $\mathbf{4 h r}$ & $\mathbf{6 h r}$ & $\mathbf{1 2 h \mathbf { ~ }}$ & $\mathbf{1 8 h \mathbf { h }}$ & $\mathbf{2 4 h \mathbf { h }}$ \\
\hline- Study & 0 & 0 & 0 & 0 & 5.6 & 26.25 & 31.87 & 7.5 & 22.5 \\
\hline- Control & 0 & 0 & 0 & 25.25 & 33.75 & 7.5 & 65.62 & 13.12 & 58.12 \\
\hline
\end{tabular}

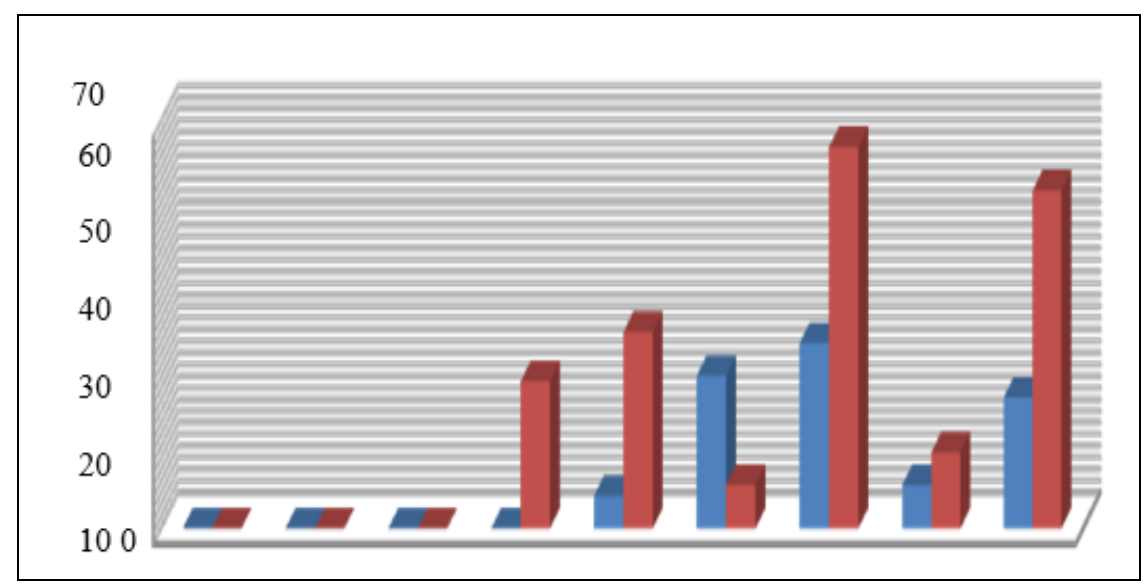

Postoperative (Hours)

Tap block with $0.5 \%$ Ropivacain reduces requirement of DICLOFENAC in study group compare to control group all the time except at 6 hour.

Table 6: Complications of TAP block

\begin{tabular}{|c|c|c|c|}
\hline Complication & $\begin{array}{c}\text { Study } \\
\text { group n=40 }\end{array}$ & $\begin{array}{c}\text { Control } \\
\text { group n=40 }\end{array}$ & $\begin{array}{c}\mathbf{P} \\
\text { Value }\end{array}$ \\
\hline Failure & 0 & 0 & 0 \\
\hline $\begin{array}{c}\text { Local anaesthetic } \\
\text { toxicity }\end{array}$ & 0 & 0 & 0 \\
\hline $\begin{array}{c}\text { Intra peritoneal } \\
\text { injection }\end{array}$ & 0 & 0 & 0 \\
\hline Bowel injury & 0 & 0 & 0 \\
\hline Hepatic injury & 0 & 0 & 0 \\
\hline
\end{tabular}

There were no any complications related to the USG guided TAP block technique in any patient.

\section{Discussion}

Inadequately controlled post-operative pain has undesirable physiological and psychological consequences such as morbidity, delayed recovery and patient dissatisfaction.

Following inguinal hernia surgery, moderate or severe pain may increase the duration of hospital stay, unexpected rehospitalization, delay in returning to normal activities and increase in associated costs.

Various methods and medications are used in postoperative pain management. The most common approach to postoperative pain relief is multimodal using NSAIDs, opioids and local infiltration of local anesthetic.

Opioids are effective for treatment of postoperative pain but can cause adverse effects such as nausea, vomiting, decreased gastrointestinal motility, respiratory depression and sedation which further increases the morbidity of the patient. Local infiltration does not relieve deep muscular pain and NSAID causes haemostasis alteration, renal dysfunction, gastrointestinal hemorrhage.

Rafi described TAP block for post-operative pain relief following abdominal surgeries ${ }^{[5]}$. Hebbard et al. who described the block under USG guided in triangle of petit ${ }^{[7]}$. Jankovic et al. found that all nerves of interest traversed the TAP at midaxillary line ${ }^{[8]}$. Good analgesia above and below the umbilicus was produced by oblique sub-costal and lateral approach to TAP block. The four-point single-shot technique combining bilateral oblique subcostal and posterior TAP block to provide bilateral analgesia was described by BØrglum et al ${ }^{[11]}$.

TAP block used in our practice leads to better VAS score despite open abdominal surgery. Study group required less diclofenac mean dose at 24 hours than control group. TAP block is highly efficacious in providing post-operative analgesia with lower VAS score upto 24 hours which has been confirmed by our as well as various studies ${ }^{[6,7]}$. Niraj at el. documented opiod sparring effect with TAP block ${ }^{[9]}$. Similar results of reduced NSAIDs use has been observed in our study, thereby reducing the incidence of NSAIDs related side effects.

Time to rescue analgesia was prolonged in our study group where TAP block was administered which was in accordance with the study of McDonnel et al. and Carney et $a l^{[10]}$.

\section{Conclusion}

1. Easy to perform, quick, safe and precise.

2. Effective as post-operative analgesic regimen for patient undergoing inguinal hernioplasty when used as a part of multimodal analgesia as it reduces pain(VAS score) and increases duration of time to first requirement of rescue analgesic drug (diclofenac).

3. It reduces total NSAID requirement (Diclofenac) in 24 hours and their related side effects.

4. Associated with minimal chances of complications.

\section{References}

1. Jenkins $J$, O'Dwyer $P$. Inguinal hernias. BMJ. 2008;336(7638):269-272.

2. Kurzer M, Kark A, Hussain S. Day-case inguinal hernia repair in the elderly: A surgical priority. Hernia. 2008;13(2):131-136.

3. Aasvang E. Chronic postoperative pain: the case of inguinal herniorrhaphy. British Journal of Anaesthesia. 2005;95(1):69-76.

4. Joshi G, Rawal N, Kehlet H. Evidence-based management of postoperative pain in adults undergoing open inguinal hernia surgery. British Journal of Surgery 2011;99(2):168-185. 
5. Rafi A. Abdominal field block: a new approach via the lumbar triangle Anaesthesia. 2001;56(10):1024-1026.

6. McDonnell J, O'e Donnell B, Tuite D, Farrell T, Power C. The regional abdominal field infiltration (R.A.F.I.) technique: computerized tomographic and anatomical identification of a novel approach to the transverses abdominis neuro-vascular fascial plane Anesthesiology 2004;101:A899.

7. Hebbard P, Fujiwara Y, Shibata Y, Royse C. Ultrasound-guided Tranversus abdominis plane (TAP) block. Anaesthesia and Intensive Care 2007;35:616-8.

8. Jankovic ZB, du feu FM, McConnel P. An anatomical study of the Tranversus abdominis plane block. Location of the lumber triangle of petit and adjacent nerves. Anaesth Analg 2009;109:981-5.

9. Niraj G, Searle A, Mathews M, Mishra V, Baban M, Kianis S, et al. Analgesic efficacy of Tranversus abdominis plane block in patient undergoing open appendisectomy. Br. J Anaesth 2009;103:601-5.

10. Carney J, McDonnel JG, Ochana A, Bhinder R, Laffey JG. The Tranversus abdominis plane block provides effective post-operative analgesia in patient undergoing total abdominal hysterectomy. Anaesth Analg 2008;107:981-5.

11. BØrglum J, Maschmann C, Belhage B, Jesen K. Ultrasound-guided bilateral dual Tranversus abdominis plane block. A new four-point approach. Acta Anaesthesiol Scand 2011;55:658-63. 